${ }^{4}$ The Research Foundation for Microbial Disease of Osaka University, Osaka, Osaka, Japan

Introduction Influenza is a potentially serious infection among hematooncology patients. The Immune system in these patients has been suppressed, and the antibody response to vaccines is supposed very poor. We conducted this study for the purpose of evaluating the immunogenicity and reactogenicity of the pandemic vaccine among immunocompromised hematooncology patients.

Materials and Methods During the 2009-2010 influenza season, 50 hematooncology patients (20 men, 30 women; mean age: $58.5 \pm 13.8$ years) received two doses of monovalent inactivated unadjuvanted vaccine. The immunogenicity of the vaccine was evaluated according to conventionally used international criteria (EMEA, 1997; FDA, 2007).

Results The geometric mean of HI titre 4 weeks after vaccination increased from a pre-vac level of 5.0 to 12.0 for the first injection, and to 21.0 for the second injection. Both the sero-conversion and protection rates were $27 \%$ for the first injection, and $46 \%$ for the second injection. Within 1 year dosages of the rituximab (anti-CD20 monoclonal antibodies) decreased sero-response rates significantly, showing that rituximab had invalidated the vaccine's efficacy. No unique adverse events were detected.

Conclusions Although the immune system in hematooncology patients has been suppressed, a two-shot influenza vaccination series showed a booster effect and achieved the EMEA criteria. Dosages of rituximab invalidated the vaccine's efficacy within one year. Therefore, optimal timing for influenza vaccination is needed for patients receiving chemotherapy.

Funding This study was supported by a research grant for Research on Emerging and Re-emerging Infectious Diseases, Ministry of Health, Labor and Welfare, Japan.

\section{P2-422 EPIDEMIOLOGY BY ZIP CODE FOR CANCER STUDY IN TEHRAN CITY}

doi:10.1136/jech.2011.142976l.52

B Ighanian. * Cancer Research of Cancer Institute, Tehran, Iran

Geographical Information System is a computerised system for managing and analysing the geographic information. It has the capability to gather, save, analyse and show the geographic information. Considering utilising of zip codes has segregation of information in residential usage, the experts of the ministry of health and medical support this method (utilising zip codes) as the best way to reach to their aims. Digitising 18 sheets of the plans of Tehran considering various methods of clarification of existent information including 700 thousand informational records, were analysed and tested by different softwares like ArcGIS, Arcview, Edvisi, Ilwis and AutoCAD. These methods contain, rechecking the information by operators and clarification of information on the postal and geographical plans by mentioned softwares. As the addresses in the files of The Office of Codifying were edited in three parts (sector, penultimate pass way, last pass way) The program firstly verifies all three fields with data bank of cancer to attribute 5 digits codes to patient's address, and then if two fields in one record are verified with data bank, 5 digits of zip code is attributed to the patient's address and finally all records which have received codes are eliminated thus these records are moved out of the comparing circle. The plans show that the most aggregated zone of cancer epidemy is Bazar and south west is the second most aggregated zone. This method can be use in several cases such as EOC GIS (Emergency Operation Center), Sustainable development, health GIS, Crime GIS, Educational management, Economical cases and any other macro managements system.

\section{P2-421 WOMEN, VIOLENCE AND TOBACCO: ARE WE MISSING AN IMPORTANT LINK?}

doi:10.1136/jech.2011.142976l.51

0 Ige, 0 Adebiyi, 0 Popoola. University of Ibadan, Ibadan, Oyo, Nigeria

Introduction The tobacco industry now aggressively targets women in order to increase its consumer base, since fewer women than men use tobacco all over the world. In response to the call by the WHO for more research aimed at understanding tobacco use among women, this study examined associations between history of domestic violence and current tobacco use among women of reproductive age.

Methods Data from the 2008 Nigeria Demographic Health Survey (NDHS), a nationally representative cross sectional survey of 33385 women aged 15-49 was analysed. Logistic regression analysis was used to assess associations between domestic violence and tobacco use. Results Only $0.9 \%$ of women were current users of tobacco in any form. Of these, $0.5 \%$ used snuff, $0.2 \%$ used cigarettes, $0.1 \%$ used chewing tobacco while $0.1 \%$ smoked pipes. The odds of tobacco use increased with experiences of severe physical violence $[\mathrm{OR}=2.9 ; 95 \%$ CI 2.2 to 3.9 ] and sexual violence [OR=2.9; $95 \%$ CI 2.0 to 4.1$]$ but not with emotional violence. Those women who had also been hurt physically by a former partner had higher odds of current tobacco use $[\mathrm{OR}=5.2 ; 95 \% \mathrm{CI} 2.1$ to 12.8]. Interestingly women who used tobacco had more often also physically hurt their husbands even when the husbands were not hurting them $[\mathrm{OR}=2.2 ; 95 \% \mathrm{CI} 1.3$ to 3.8].

Conclusion Experiencing violence affects the psychological health status and may explain susceptibility to tobacco use in women. In the spirit of World No Tobacco Day 2010 tobacco control strategies must recognise the psychosocial environment of tobacco users and also address domestic violence for sustained impact.
${ }^{1} \mathrm{M}$ Jergovic, ${ }^{*}{ }^{1,2} \mathrm{D}$ Puntaric, ${ }^{2} \mathrm{M}$ Miskulin. ${ }^{1} \mathrm{Dr}$. Andrija Stampar Institute of Public Health, Zagreb, Croatia; ${ }^{2}$ Department of Public Health, Josip Juraj Strossmayer University of Osijek, School of Medicine, Osijek, Croatia

Aim To determine metal and metalloid exposure in the people of east Croatia exposed to heavy and moderate fighting during the 1991-1995 war in Croatia.

Methods The concentrations of 18 elements, that is, aluminium $(\mathrm{Al})$, arsenic (As), barium (Ba), cadmium (Cd), chromium (Cr), copper $(\mathrm{Cu})$, iron $(\mathrm{Fe})$, manganese $(\mathrm{Mn})$, molybdenum (Mo), niobium $(\mathrm{Nb})$, nickel $(\mathrm{Ni})$, lead $(\mathrm{Pb})$, titanium $(\mathrm{Ti})$, uranium $(\mathrm{U})$, vanadium $(\mathrm{V})$, tungsten $(\mathrm{W})$, zinc $(\mathrm{Zn})$ and zirconium $(\mathrm{Zr})$, reported to be associated with military operations, were determined in hair, serum and urine samples using inductively-coupled plasma mass spectroscopy (ICP-MS). A total of 391 participants from east Croatian areas of heavy and moderate fighting were included in this biomonitoring cross-sectional study.

Results Higher concentrations of the selected elements associated with fire arms (Al, As, Ba, Cd, Cr, Cu, Mn, Mo, Ni, Pb, U, V, W, Zn i $\mathrm{Zr}$ ) were determined in one or more samples of study participants from east Croatia as compared with literature data available.

Conclusion Study results revealed high concentrations of the selected elements in biological samples of the study population from east Croatia, pointing to the need of comprehensive risk assessment and extensive monitoring of metal and metalloid exposure in the populations living in former conflict zones, emphasising the role of biomonitoring through ecologic and preventive activities. 